\title{
Adaptive Virtual Machine Provisioning in Elastic Multi-tier Cloud Platforms*
}

\author{
Fan Zhang ${ }^{1}$, Junwei $\mathrm{Cao}^{2,3}$, Hong $\mathrm{Cai}^{4}$, James J. Mulcahy ${ }^{5}$, Cheng Wu ${ }^{1,3}$ \\ ${ }^{I}$ National CIMS Engineering and Research Center, Department of Automation \\ Tsinghua University, Beijing 100084, P. R. China \\ ${ }^{2}$ Research Institute of Information Technology, Tsinghua University, Beijing 100084, P. R. China \\ ${ }^{3}$ Tsinghua National Laboratory for Information Science and Technology, Beijing 100084, P. R. China \\ ${ }^{4}$ IBM China Software Development Lab \\ ${ }^{5}$ Florida Atlantic University, Boca Raton, Florida, USA
}

\begin{abstract}
Virtual machines are allocated on demand in virtualized cloud platforms to provide flexible and reliable services. The major difficulty lies in satisfying the conflicting objectives of reducing response time while lowering resource costs. In this paper, a mathematical multi-tier framework for virtual machine allocation is proposed, which can be used to capture the performance of the cloud platform. We first use simulations to derive virtual resource allocation policies, and later use real benchmarking applications to verify the effectiveness of this framework. Experimental results show that the model can be simply and effectively used to satisfy the response time requirement as well as lowering the cost of using the virtual machine resources.
\end{abstract}

Key words: Cloud platform; dynamic resource provision; multi-tier applications; and virtual machines.

\section{Introduction}

\subsection{Research background}

Cloud computing allocates Virtual Cluster (VC) resources in software as a service (SaaS)[1], platform as a service (PaaS)[2], and infrastructure as a service (IaaS)[3] applications. A virtual cluster [4] is an organized heterogeneous Virtual Machine (VM) hosting platform, which is used in cloud platforms to provide flexible and reliable services. Examples of virtual machine software include VMware[5] and Xen[6]. The VMs are allocated on demand, which satisfies user requirements for response time while lowering the cost of using the resources.

Dynamic resource provisioning [7], which has been widely used in internet hosting platforms, has proven to be useful in handling multiple time-scale workloads. However,

Manuscript submitted to IEEE NAS 2010 on March 12, 2011. All rights reserved. This work is performed when Fan Zhang was an intern student in IBM China CDL supported by 2010-2011 IBM Ph.D. Fellowship. Corresponding author is Junwei Cao. He can be reached via: jcao@tsinghua.edu.cn this kind of dynamic provisioning in previous work has been more commonly based upon physical resource allocation, which is not flexible enough for the effective delivering of services.

Unlike other resources, VMs are flexibly deployed on physical machines, which can be automatically generated for different applications. For example, some scientific applications such as gene expression and transferring demand a lot of CPU resources, while other real-time transaction applications and interactive online games are memory and network-intensive. Though traditional physical capacity provisioning has long been used, overprovisioning or under-provisioning has been a common difficulty for most resource vendors.

The advent of cloud computing, which takes full advantage of virtualization technology, potentially solves this difficulty. The flexibility of our proposed adaptive resource provisioning is threefold:

(1) The flexibility of allocating virtual resources: we can dynamically create and/or destroy a VM from a resource pool using a simple command. This advantage can be used to handle the problems in resource overprovisioning and/or under-provisioning.

(2) The flexibility of handling data or communication locality: we can easily deploy those VMs that have the most frequent communication or interaction in such a way that they are physically close to each other. This advantage saves the communication cost noticeably.

(3) The flexibility of migrating services: virtual resources used to provide reliable services can use service migration to re-organize service hosting platforms in order to maximize the utilization of resources, which can dramatically improve operational costs.

Given the above advantages from the use of adaptive resource provisioning, we propose the design of a virtualized resource allocation framework using the cloud platform, which allocates VMs on demand in order to 
provide services, as well as minimizing the cost of using those virtual resources.

\subsection{Motivation}

One of the major difficulties of virtual resource allocation is how to provide proper policies at different times to satisfy different user demands. This difficulty lies in the unpredictable execution time, variable operational costs, and frequently fluctuating workloads [8]. These aspects require a proper model to capture the characteristics of highly complex systems.

Model-View-Control (MVC), an architectural design pattern widely used on internet hosting platforms, is also supported in Windows Azure[9] and other cloud platforms. This design pattern is unique to the new proposed paradigm in that full virtualization is employed in order to achieve higher reliability and flexibility. Based on a typical MVC architecture, we model our application as a multi-tier queuing network to leverage virtual resource provisioning.

The inputs of this model are the cost of using each VM at each tier, the transferring probability that a request should be forwarded to the next or previous tier, and most importantly, the inter-arrival time of the workloads. The output is the optimal number of VMs allocated for each tier needed to achieve a reasonable response time and minimized total resource costs. We analyze the relationship of these parameters and show its derivation based on queuing theory [10].

\subsection{Contributions of this work}

In this paper, we focus on the mathematical formation and optimization of the model, and show how the above goals can be satisfied in a simple yet applicable way. The major contributions of this work are described as follows:

(1) Derives response time through mathematic formulation: we establish a direct relationship between a workload and average response time could be caused by this workload through a queuing model. The relationship can be used to determine the number of VMs we need to satisfy user demands while minimizing the cost of using these VMs.

(2) Extends traditional single size VM scheduling problems: our model includes two kinds of VM instances (large and small) that have a different number of CPUs and memory capacities to provide different computing capabilities. This extension is much more applicable in cloud platforms such as Amazon EC2 [23] and Eucalyptus [24].

(3) Carries out benchmarks using the Rice University Bidding System (RUBiS) on IBM X3950 servers with different sizes of VMs, to verify the correctness and efficiency of our proposed model. This model is also tested with simulations to make comparisons.
The rest of this paper is organized as follows: We introduce the related work in section 2. System architecture, including our virtualized cloud platforms and queuing network model are introduced in section 3. The virtual resource allocation framework is introduced in section 4 . We describe experimental studies using both simulation and real benchmarks to verify our proposed method in section 5. We conclude this work and propose the future research direction in section 6 .

\section{Related work}

Modeling a single tier of servers is first proposed in [11], where four queues are adopted in a model web server. Two are used for the web servers and the remaining two are used for modeling communication networks. In [12], modeling CPU, memory and disk bandwidth is suggested for performance prediction in web servers. Modeling replicated a single tier application using a $\mathrm{G} / \mathrm{G} / 1$ queuing network is also discussed in [13]. Using an M/M/1 queuing model to calculate response times of web requests are proposed in [14]. The throughput of web servers using a queuing network combined with Markov chain is suggested in [15]. These works discuss a single tier server solution, and later a multi-tier platform. We differentiate our work by using multi-tier internet hosting platforms.

There is existing prior work using queuing networks to model multiple tier internet hosting platforms. An $\mathrm{M} / \mathrm{G} / 1 / \mathrm{PS}$ model is proposed in [16] to provide servers on application tiers. A G/G/N queuing system with $\mathrm{N}$ servers for an e-commerce application model is discussed in [17]. An M/GI/1/PS model for an e-commerce application is also suggested in [18] to use a single queue to model multitier systems. The similarity of these works is that they only model the bottleneck aspects of the servers, which cannot capture the global characteristics of an entire system. In our model, the framework covers all the parts of a multitier system, including capturing bottleneck issues with the use of load balancing techniques.

A queuing network based analytical model is proposed to model multi-tier internet hosting platforms in [19]. An enhanced model of this work can be used to deal with load balancing, handling concurrency limit, and multiple session classes at each tier. The improvement of this work is suggested in [20], where a combination of predictive and reactive methods is used to determine when to provide virtual resources. [20] also uses a queuing network model to determine how much virtual resource to provide. Our work is different from these works in that it not only takes the cost of using virtual resources into consideration, but also includes a framework to schedule small and large VMs upon user demand. These different-sized VM provisioning techniques are commonly seen in Eucalyptus [23], Amazon EC2 [24], and other cloud platforms. 
Recently, cloud computing [21] has become a popular research topic in which computational resources are virtualized based on user demand. Buyya's work in [22] has a similar motivation with ours with respect to cost minimization ', and they use CloudSim [27] for simulating data centers. We differentiate our work using adaptive virtual machines provisioning on virtualized cloud computing platforms.

We propose a quantitative framework to analyze the VM scheduling problem, in which virtual clusters are used in each tier of a queuing network to provide services on demand based on different workloads.

\section{System architecture overview}

In this section, we introduce our virtualized cloud platform using a queuing network. We then formulate this virtual resource scheduling problem as a constrained integer optimization problem.

\subsection{Virtualized cloud platforms}

Physical Cluster 1 Physical Cluster 2 Physical Cluster 3

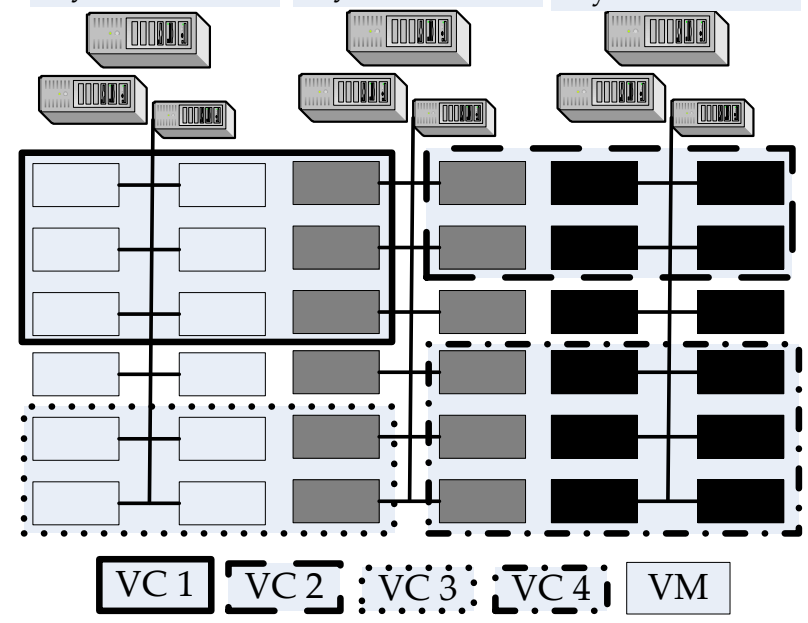

Fig 1. Four virtual clusters built over 3 physical clusters. Each physical cluster consists of a number of interconnected servers, represented by the three servers. Each physical cluster contains 12 virtual machines, represented by the rectangular boxes with 3 different shadings. The virtual machines are implemented on the servers (physical machines). Each virtual cluster can be formed with either physical machines or VMs hosted by multiple physical clusters. The boundaries of the virtual clusters are shown with 4 dot/dash-line boxes. The provisioning of VMs to a virtual cluster can be dynamically based upon user demands.

In a virtualized cloud platform, various physical clusters are utilized to provide computational resources, such as CPU and memory allocations. A physical cluster contains many interconnecting physical nodes. VMs are deployed on these physical nodes based on their CPU or memory configuration. In Fig. 1, we show 3 physical clusters. Each physical cluster contains 3 physical nodes with 12 VMs deployed in each physical cluster.

Virtual clusters are used to partition or reorganize the VMs based on the computational capacity of each VM, the requirement of different application scenario, or more specifically, different workloads. The purpose is to provide an automatic scaling or shrinking mechanism to improve utilization. This mechanism gives customers reliable response times and service availability. It also allows for flexible resource provisioning on demand, which can reduce the cost for using the resources. It benefits vendors in that the resources are allocated as needed which takes utilization into account, providing more effective services.

Based on the resource provisioning principles that many cloud service vendors use, different VMs are predeployed with different resources. For example, some VMs are deployed with $1 \mathrm{CPU}$ core with $1 \mathrm{~GB}$ memory (small VMs). Some are deployed with 2 CPU cores with 2 GB memory (large VMs). This mechanism is shown in Fig. 2.

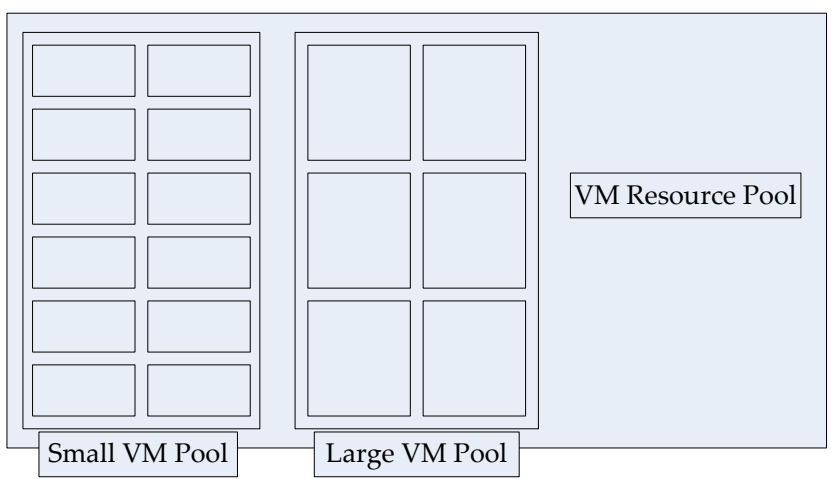

Fig. 2. VM resource pool with VMs of different computational resource. VMs in small/large VM pool are equipped with $1 / 2 \mathrm{CPU}$ cores with $1 / 2 \mathrm{~GB}$ memories. Different numbers of VMs in small/large VM pool are scheduled into virtual clusters to provide services on demand.

\subsection{Multi-tier queuing network model}

We describe our application specific to multi-tier hosting platforms in Fig. 3. To simplify the illustration, we use a typical and widely used $J$-tier platform as an example. The model in our experiment is a queuing network with $J$ queues. Each queue corresponds to one tier that handles the inter-arrival requests. There are $n$ sessions concurrently generating requests. Each request that arrives at tier $j(j \in[1, J-1])$ should either proceed into the $j+1$ tier, with a probability $P_{j}$, or return to the $j-$ 1 tier, with a probability $1-P_{j}$. The requests that arrive at the $J^{\text {th }}$ tier will be returned to the $(J-1)^{\text {th }}$ tier with a probability of 1 . Thus $P_{J}=0$. 


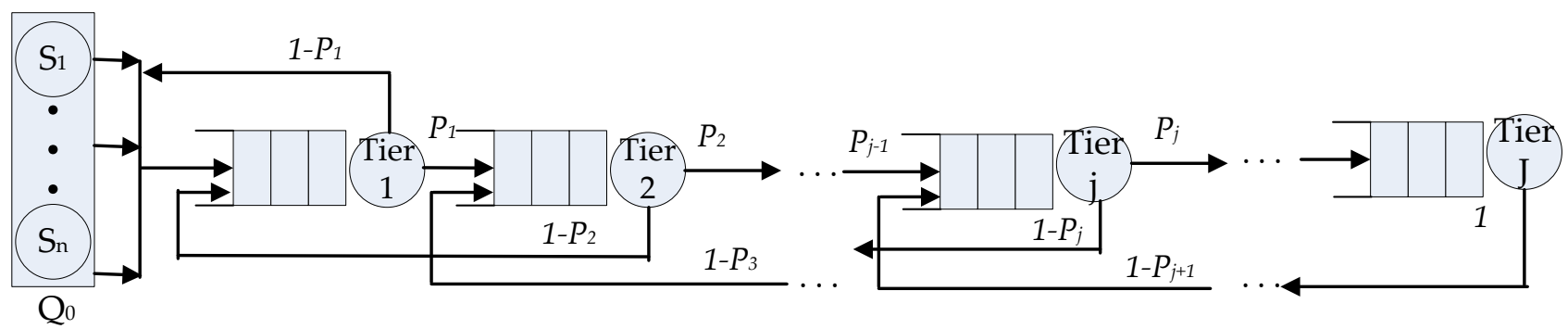

Fig. 3. A multi-tier application hosting platform with $n$ sessions generating requests. Each tier is modeled as a queuing system. Any request arriving at the $j^{\text {th }}$ tier either proceeds into the $(j+1)^{\text {th }}$ tier with a probability denoted by $P_{j}$, or returns back to the $(j-1)^{\text {th }}$ tier with probability $1-P_{j}$. All requests arriving at the $J^{\text {th }}$ tier (the last tier) should return to the $(J-1)^{\text {th }}$ tier.

Our scheduling target is to achieve an appropriate average response time for customers as well as reducing the costs of using those virtual resources. The detailed formulation of this problem is elaborated in the following section.

To benefit readers, Table 1 introduces the major symbols, concepts, definitions and explanations that will be used in the following sections.

Table 1. Symbols and definitions

\begin{tabular}{|c|c|}
\hline$i(i-1)$ & The current/previous stage \\
\hline$j(J)$ & Number of current/total tiers \\
\hline$N_{j}^{S}(i) / N_{j}^{L}(i)$ & $\begin{array}{l}\text { Number of small/large VMs used of the } \\
j^{\text {th }} \text { tier in the } i^{\text {th }} \text { stage }\end{array}$ \\
\hline$\mu_{j}^{S}(i) / \mu_{j}^{L}(i)$ & $\begin{array}{l}\text { Avg. service rate of small/large VM of } \\
\text { the } j^{\text {th }} \text { tier in the } i^{\text {th }} \text { stage }\end{array}$ \\
\hline$\rho_{j}^{S}(i) / \rho_{j}^{L}(i)$ & $\begin{array}{c}\text { Avg. arriving-service ratio of } \\
\text { small/large VM }\end{array}$ \\
\hline$A S T_{j}^{S} / A S T_{j}^{L}$ & $\begin{array}{l}\text { Avg. staying time of each request in } \\
\text { small/large VM of the } j^{\text {th }} \text { tier }\end{array}$ \\
\hline$A A R_{j}\left(\lambda_{j}(i)\right)$ & Avg. arrival rate of the $j^{\text {th }}$ tier \\
\hline$\lambda_{j}^{\prime}(i)$ & Avg. arrival rate of each VM in the $j^{\text {th }}$ tier \\
\hline $\begin{array}{l}A A R_{j-1, j} \\
A A R_{j+1, j}\end{array}$ & $\begin{array}{l}\text { Avg. arrival rate of the } j^{\text {th }} \text { tier from the } \\
(j-1)^{t h} /(j+1)^{t h} \text { tier }\end{array}$ \\
\hline $\begin{array}{l}A D R_{j, j-1} \\
A D R_{j, j+1}\end{array}$ & $\begin{array}{l}\text { Avg. departure rate of the } j^{\text {th }} \text { tier to the } \\
\qquad(j-1)^{t h} /(j+1)^{t h} \text { tier }\end{array}$ \\
\hline$A D R_{j}$ & Avg. departure rate of the $j^{\text {th }}$ tier \\
\hline$A S T_{j}$ & Avg. staying time of request in the $j^{\text {th }}$ tier \\
\hline$C_{j}$ & Concurrency limit of VM in the $j^{\text {th }}$ tier \\
\hline$R(i)$ & Requests that are generated during stage $i$ \\
\hline$P_{j}$ & $\begin{array}{l}\text { The probability a request generated from } \\
\text { tier } j \text { proceeds into tier } j+1 \text {. }\end{array}$ \\
\hline$\Theta_{S} / \Theta_{L}$ & Available number of small/large VM \\
\hline
\end{tabular}

The relationship of these symbols and their roles in this work are introduced in the followed section.

\section{Virtualized resource scheduling}

\subsection{Modeling of tier structure}

Suppose in the $i^{\text {th }}$ stage, the number of small/large VM of the $j^{\text {th }}$ tier is denoted as follows in E.Q. 2:

$$
\begin{aligned}
& N_{j}^{S}(i)=N_{j}^{S}(i-1)+\Delta N_{j}^{S}(i-1) \\
& N_{j}^{L}(i)=N_{j}^{L}(i-1)+\Delta N_{j}^{L}(i-1)
\end{aligned}
$$

As shown in Fig. 3, the expected inter-arrival rate of requests is denoted by $\lambda_{j}(i)$, and the inter-arrival rate for each queue is denoted by:

$$
\lambda_{j}^{\prime}(i)=\lambda_{j}(i) /\left[N_{j}^{S}(i)+N_{j}^{L}(i)\right]
$$

$\lambda_{j}(i)$, which is also called $A A R_{j}$, is composed of two parts as shown in Fig. 3. The first part is the requests coming from the previous tier, namely the $(j-1)^{\text {th }}$ tier, which is denoted by $A A R_{j-1, j}$, and the second part is the requests that are coming from the following tier, namely the $(j+1)^{\text {th }}$ tier, as denoted by $A A R_{j+1, j}$. We write this as follows:

$$
\lambda_{j}(i)\left(A A R_{j}\right)=A A R_{j-1, j}+A A R_{j+1, j}(j \in[1, J-1])
$$

$A A R_{0,1}$ is the inter-departure rate of $\mathrm{Q}_{0}$. In the $J^{\text {th }}$ tier, we have

$$
\lambda_{J}(i)\left(A A R_{J}\right)=A D R_{J-1, J}
$$

Each VM can be viewed as an $\mathrm{M} / \mathrm{M} / 1 / \mathrm{C} / \infty / \mathrm{FIFO}$ queuing system [10]. $C_{j}$ is the concurrency limit of each VM. Thus we can calculate the average staying time for each request in small/large VM of the $j^{\text {th }}$ tier by:

$$
\begin{gathered}
A S T_{j}^{S}=\frac{1}{\mu_{j}^{S}(i)-\lambda_{j}^{\prime}(i)}-\frac{C_{j} \rho_{S}{ }^{C_{j}+1}}{\lambda_{j}^{\prime}(i)\left(1-\rho_{S}{ }^{C_{j}}\right)}, \rho_{j}^{S}(i)=\frac{\lambda_{j}^{\prime}(i)}{\mu_{j}^{S}(i)} \\
A S T_{j}^{L}=\frac{1}{\mu_{j}^{L}(i)-\lambda_{j}^{\prime}(i)}-\frac{C_{j} \rho_{L}^{C_{j}+1}}{\lambda_{j}^{\prime}(i)\left(1-\rho_{L}{ }^{C_{j}}\right)}, \rho_{j}^{L}(i)=\frac{\lambda_{j}^{\prime}(i)}{\mu_{j}^{L}(i)}
\end{gathered}
$$

Based on the above analysis, the average departure rate of the $j^{\text {th }}$ tier is

$$
A D R_{j}=N_{j}^{S}(i) / A S T_{j}^{S}+N_{j}^{L}(i) / A S T_{j}^{L}
$$


Next, we can derive the average staying time of each request in the $j^{\text {th }}$ tier as:

$$
A S T_{j}=1 / A D R_{j}
$$

Based on our model in Fig. 4, the average arrival rate of the $(j+1)^{\text {th }}$ tier from the $j^{\text {th }}$ tier is denoted by

$$
A A R_{j, j+1}=A D R_{j} * P_{j}
$$

Similarly, the arrival rate of the $(j-1)^{\text {th }}$ tier from the $j^{\text {th }}$ tier is denoted by

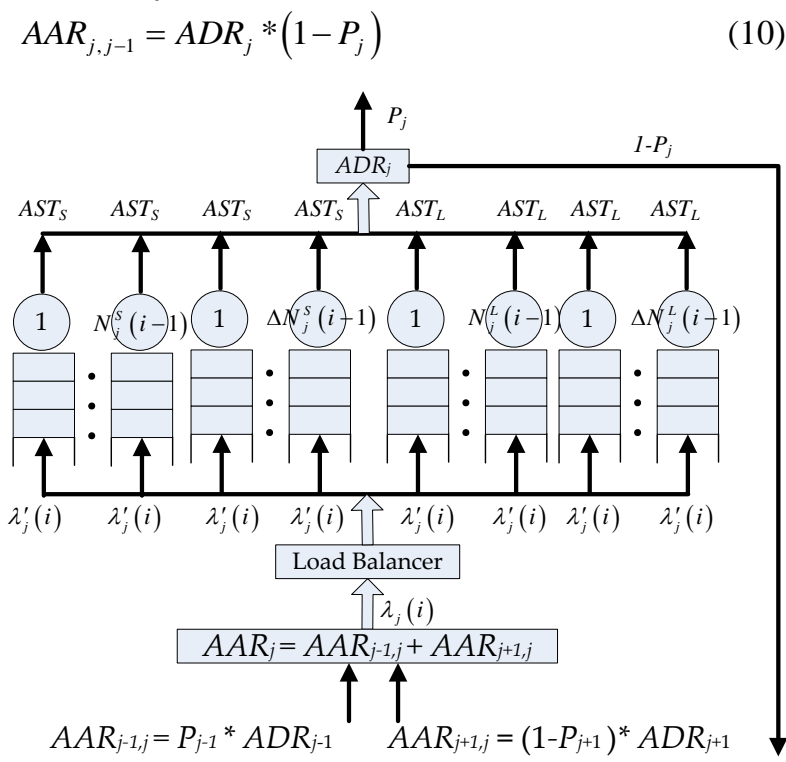

Fig. 4. The inner structure of the $j^{\text {th }}$ tier at the $i^{\text {th }}$ stage: Each VM is modeled as an individual queuing system. A load balancer is used to fairly distribute the incoming workloads to the VMs. Our target is to find an optimal number of large and small VMs in each layer at each stage in order to satisfy the response time demands in SLA.

\subsection{Implementation procedure consideration}

Given the number of large and small VMs of the $j^{\text {th }}$ tier at the $i^{\text {th }}$ stage as demonstrated above, we can calculate the expected average response time at this stage as follows.

(1) Analyze the $J^{\text {st }}$ tier:

Based on E.Q. (7), $A D R_{J}$ can be represented as a function of $\lambda_{J}(i)$, which is initially unknown. We also can derive the average staying time of each request at the $J^{\text {st }}$ tier as $A S T_{J}=1 / A D R_{J}$ based on E.Q. (8). In $A S T_{J}$, we still have one unknown variable: $\lambda_{J}(i)$.

(2) Analyze the $(J-1)^{\text {th }}$ tier:

Since $\lambda_{J-1}(i)=A A R_{J-2, J-1}+A A R_{J, J-1}=A A R_{J-2, J-1}+A D R_{J}$, we have two unknown variables: $A A R_{J-2, J-1}$ and $\lambda_{J}(i)$. Now we can calculate $A D R_{J-1}$ based on (7), which also has the same two unknown variables. Since $\lambda_{J}(i)=A D R_{J-1} * P_{J-1}$, we replace $\lambda_{J}(i)$ with $A D R_{J-1} * P_{J-1}$ in $A D R_{J-1}$. In this way, $A D R_{J-1}$ becomes a function of $A A R_{J-2, J-1}$, thus there is only one unknown variable at the end of this step. We can also derive $A S T_{J-1}=1 / A D R_{J-1}$ as shown in (1), which also has one unknown variable.

(3) Analyze the $j^{\text {th }}$ tier. $j$ is valued sequentially from $J-2$ to 1:

We repeat the steps in (2) and calculate $A S T_{j}$, which is a function of an unknown variable $A A R_{j-1, j}$. When we come to the $1^{\text {st }}$ tier, we have $A A R_{0,1}$ as the average arrival rate of the request generating in clients, which is a known variable in our experiment. In this way, we can calculate the value of $A D R_{l}$ and $A S T_{l}$. If $A A R_{0,1}$ is unknown beforehand, we can use a method such as Kalman filter or neural network to predict the workload of the first tier.

(4) Analyze the $j^{\text {th }}$ tier: $j$ is valued sequentially from 2 to $J$.

In the previous step, we obtain the value of $A D R_{l}$. We can calculate $A A R_{1,2}$ as $P_{1} * A D R_{l}$. Since $A S T_{2}$ is a function of $A A R_{1,2}$, we can calculate $A S T_{2}$. Similarly, we can go through each tier to get $A S T_{j} . j$ is sequentially valued from 2 to $J$.

(5) Calculate the average response time:

Suppose there are $R(i)$ requests processed at the $i^{\text {th }}$ stage. Based on Fig. 3, we use $\operatorname{Num}\left(R_{j}(i)\right)$ to denote the number of requests that are processed sequentially from the $1^{\text {st }}$ tier to the $j^{\text {th }}$, and then return to the $1^{\text {st }}$ tier until reaching $\mathrm{Q}_{0}$. Notice that these requests do not visit the $(j+1)$ tier. We use TraverseTime $\left(R_{j}(i)\right)$ as the average traverse time for each of the above requests. The total average response time can be calculated as shown in E.q. (11).

Table 2 is used to show the relationship between $j$, $\operatorname{Num}\left(R_{j}(i)\right)$ and TraverseTime $\left(R_{j}(i)\right)$, which will then be used to determine the average response time.

\begin{tabular}{|c|c|c|}
\hline$j$ & $\operatorname{Num}\left(R_{j}(i)\right)$ & TraverseTime $\left(R_{j}(i)\right)$ \\
\hline 1 & $R(i)\left(1-P_{l}\right)$ & $R(i)\left(1-P_{1}\right) * A S T_{1}$ \\
\hline 2 & $R(i) P_{1}\left(1-P_{2}\right)$ & $R(i) P_{l}\left(1-P_{2}\right) *\left(A S T_{2}+2 * A S T_{1}\right)$ \\
\hline--- & --- & --- \\
\hline$j$ & $R(i) P_{1} P_{2^{---}} P_{j-l}\left(1-P_{j}\right)$ & $R(i) P_{1} P_{2^{---}} P_{j-1}\left(1-P_{j}\right) *\left(A S T_{j}+2 * A S T_{j-1}+\ldots+2 * A S T_{1}\right)$ \\
\hline--- & --- & --- \\
\hline$J-1$ & $R(i) P_{1} P_{2^{---}} P_{J-2}\left(1-P_{J-1}\right)$ & $R(i) P_{l} P_{2^{---}} P_{J-2}\left(1-P_{J-1}\right) *\left(A S T_{J-1}+2 * A S T_{J-2}+\ldots+2 * A S T_{1}\right)$ \\
\hline$J$ & $R(i) P_{1} P_{2^{---}} P_{J-2} P_{J-1}$ & $R(i) P_{l} P_{2^{---}} P_{J-l}\left(1-P_{J}\right) *\left(A S T_{J}+2 * A S T_{J-1}+\ldots+2 * A S T_{1}\right)$ \\
\hline
\end{tabular}

Table 2. Relationship of the number of tier and traverse time 
The average response time is calculated as:

$$
\begin{gathered}
\sum_{j=1}^{J} \text { TraverseTime }\left(R_{j}(i)\right) / R(i) \\
=\sum_{j=1}^{J}\left(\prod_{k=0}^{j-1} P_{k}\right) *\left(1-P_{j}\right) *\left(2 * \sum_{l=1}^{j} A S T_{l}-A S T_{j}\right)
\end{gathered}
$$

We define $P_{0}=1$, which means the requests generated from the sessions $\left(\mathrm{Q}_{0}\right)$ are bound to arrive at the $1^{\text {st }}$ tier.

Suppose the cost of using a small/large VM is denoted by $\operatorname{Cost}_{S} / \operatorname{Cost}_{L}$ respectively. Then the total cost of the $i^{\text {th }}$ stage is:

$$
\operatorname{Cost}(i)=\operatorname{Cost}_{S} * \sum_{j=1}^{J} N_{j}^{S}(i)+\operatorname{Cost}_{L} * \sum_{j=1}^{J} N_{j}^{L}(i)
$$

Our virtual resource allocation problem is converted to a constrained optimization problem as below:

$$
\begin{gathered}
\operatorname{Min}_{N_{j}^{S}(i), N_{j}^{L}(i)}(\operatorname{Cost}(i)) \\
\sum_{j=1}^{J}\left(\prod_{k=0}^{j-1} P_{k}\right) *\left(1-P_{j}\right) *\left(\prod_{l=1}^{j}\left(2 * A S T_{l}-A S T_{j}\right)\right)<S L A \\
\sum_{j=1}^{J} N_{j}^{S}(i) \leq \Theta_{S} \\
\sum_{j=1}^{J} N_{j}^{L}(i) \leq \Theta_{L}
\end{gathered}
$$

$\Theta$ is the resource pool as shown in Fig. 2. $\Theta_{S}$ and $\Theta_{\mathrm{L}}$ denote the available number of small and large VMs respectively.

\subsection{Parameter estimation}

Based on our previous analysis, we must evaluate the parameters $P_{j}(j \in[1, J-1]), \mu_{S} / \mu_{L}$ and $\operatorname{Cost}_{S} /$ Cost $_{L}$ in order to evaluate the cost and average response time.

The transferring probability $P_{j}$ can be estimated in an experiment by calculating the average ratio of the number of requests proceeding into the $P_{j+1}$ tier to the total number of requests that arrived at tier $j$ in the previous stage. This value is easily monitored offline based on specific application scenarios.

$\mu_{j}^{S}(i) / \mu_{j}^{L}(i)$ can be estimated beforehand. As previously introduced, 1(2) CPU and 1(2) GB memories are allocated to small or large VMs, respectively. We also perform offline experiments to decide the average service rate based on a certain workload as a test. Networking latency is also included in these offline tests. Concurrency limits of small and large VMs are preset based on the servers we are using.

We estimate the cost of using the devices based on the price list found on Amazon's EC2 website. The granularity of analytical time span in our experiment is 1 minute, thus we set the cost of using a small or large VM as $\$ 0.0127$ and $\$ 0.0287$ per minute, respectively. This figure is used by combining the price of Amazon's current hourly costs and storage costs.

\section{Experimental studies}

There are two parts to our experiments. First, we use SimEvents (from Simulink) to develop event-based models of queuing networks to evaluate service times for small/large VMs in both web and application tiers. We use Matlab to calculate the response times based on our introduced formula to predict performance. The parameters, such as transferring probabilities, are derived from direct observations of RUBiS [25], an auction site similar to eBay, where transactions of bidding, buying, and selling are used to conduct real transactions. We then run RUBiS benchmark with the same parameters to compare the results.

Apache 2.0.55 is used as a web server to handle requests as a load balancer as shown in Fig. 4. We use tomcat 5.5 as an application server to deploy servlet applications, and connect to a MySQL database server. Our applications are deployed on OpenSUSE 11.

The experiments are carried out on an IBM X3950, with a CPU of 16 cores and memory of 24 GBytes. We virtualized one small and one large VM for the web servers. We also virtualized four small and two large VMs for application servers. Since the database tier is difficult to cluster, we used one large VM as database tier in order to process requests effectively.

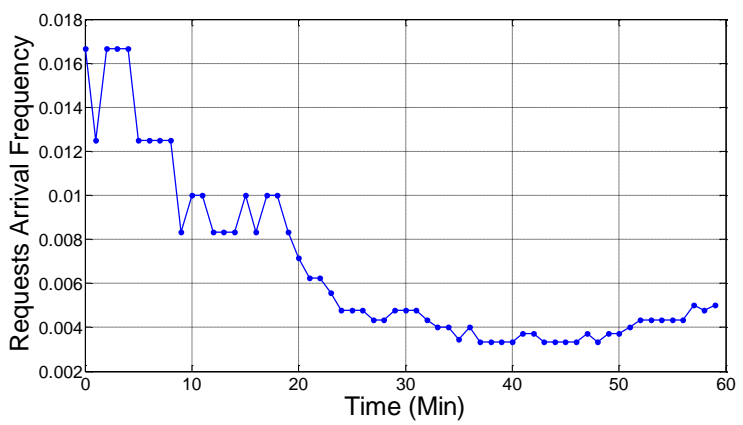

Fig. 5. The workloads generated for benchmarking. We use the inter-arrival time of requests over one hour.

Our workloads are generated based on a web trace from the 1998 Soccer World Cup site [26]. We traced average arrival times over a sixty-minute duration as shown in Fig. 5. The units of the y-axis measures arrival frequency.

In Fig. 6, we demonstrate our experimental results using different numbers of VMs as the workloads increased. We can see that the increased number of VMs can capture the characteristics of such workloads.

We further illustrate our analytical results in Fig. 7. The blue dashed line is the predicted response time from our 
queuing network model as proposed in section 4, and the black solid line denotes the measured response time using the RUBiS benchmark.

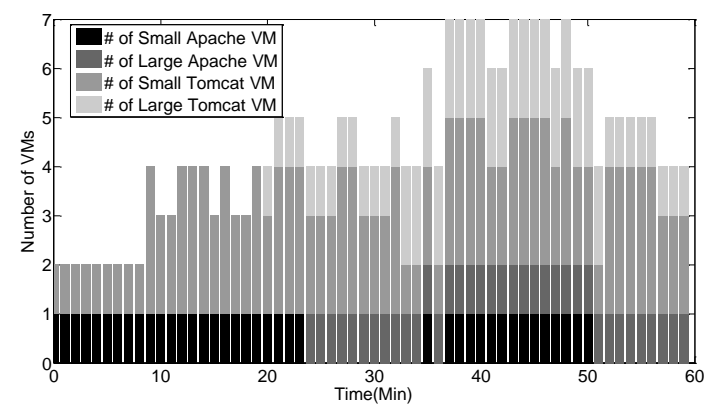

Fig. 6. The number of VMs used for web and application tier over the experiments.

The response time to satisfy the service level agreement (SLA) is set to be 10 seconds. We can see from the onehour experiment that we can satisfy the requirement with a probability close to $98 \%$. The only time this surpasses the SLA is in the 23rd minute, which is an acceptable variance.

Results show that our proposed model can be used to capture the response time, which can also be used to judge the number of VMs that should be used to properly meet the response time requirements.

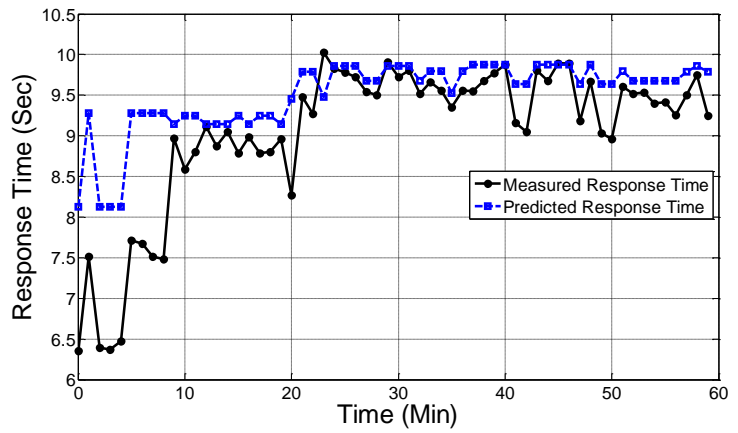

Fig. 7. Comparative results between predicted response times using the proposed queuing network model and measured response times using RUBiS in real transactions

We further compare our model with a commonly used experience-based, utilization-oriented method. It conducts VM scheduling as follows:

This method ensures that the average utilization rate of all the VMs of each tier is close to $75 \%$. If the value surpasses 95\%, a large VM is initiated (if such VM is available), else if the value is less than $95 \%$ and larger than $85 \%$, a small VM is added if one is available. Similarly, if the value is less than $45 \%$, a large VM is removed if one is running, otherwise if the value is less than $60 \%$ and larger than $45 \%$, a small VM is removed if possible. At least one small VM should be running in each tier.
This method conducts initiation and removal of VMs at the beginning of each new stage while considering the utilization of the previous stage. This method is noticeably cost-aware. The comparative results of response times and costs are shown in Fig. 8 and Fig. 9.

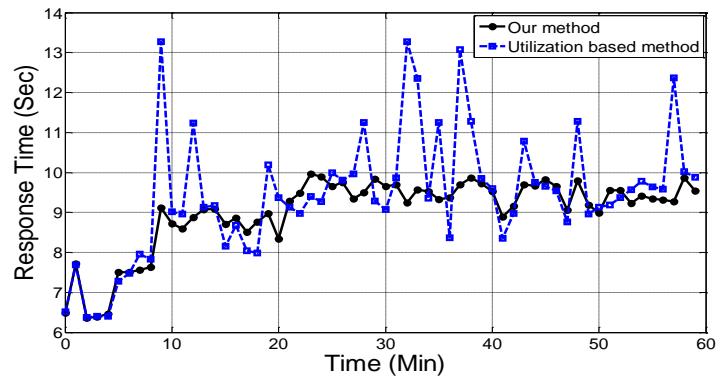

Fig. 8. Comparative results of response times between our method and utilization-based method

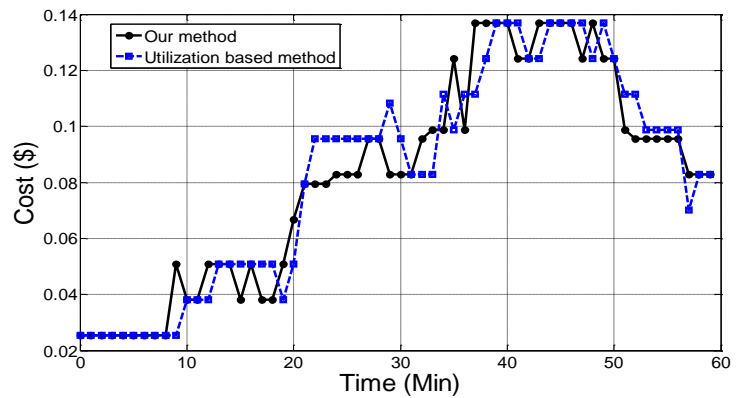

Fig. 9. Comparative results of costs between our method and utilization based method

The advantage of our proposed method is illustrated in Fig 8 and 9. From the above comparative results, we can see that utilization-based methods can also handle the workload variations. However, it cannot be agile enough to provide the proper number of VMs in order to meet the response based on time demands. From the perspective of cost, this method uses fewer resources than our proposed method, sometimes at the cost of violating the SLA.

\section{Conclusions and future work}

We first conclude our major contributions in this work and then suggest two possible directions to extend this work.

\subsection{Conclusions of this work}

In this paper, we have proposed a mathematical model for capturing the characteristics of a virtualized cloud platform using multiple virtual machine instances, and then converted this model into a constrained integer programming problem. The model can directly derive the relationship between the inter-arrival rate of requests and the average response time of requests. We first used a simulation to depict the described relationships, and then 
benchmarked our methods using RUBiS, a real transaction web site using VMs on an IBM X3950. Experimental results show that the model can be used to appropriately satisfy the response time requirements as well as reducing the costs of using those virtual machines.

\subsection{Our future work}

For further research, we suggest extending the work in the following two directions:

(1) Customized virtual resource provisioning. We propose allocations of small and large VMs based on a fixed CPU and memory settings. This can be further extended by using flexible resource provisioning principles to satisfy CPU-intensive or memory-intensive applications. To this end, we should reconsider the costs, including service deployment, starting up and shutting down of services, et cetera.

(2) Building of useful tools to serve for larger virtualized cloud platform. The Matlab simulation used to analyze the optimal number of virtual resources in our experiment should be packaged into software toolkits in order to make it available for larger virtualized cloud platforms. Our experimental software can be tailored and prototyped to this end.

\section{Acknowledgements}

This work is supported by National Science Foundation of China (grant No. 60803017) and Ministry of Science and Technology of China under National 973 Basic Research Program (grants No. 2011CB302505 and No. 2011CB302805) and National 863 High-tech R\&D Program (grants No. 2008AA01Z118 and No. 2008BAH32B03). We also thank IBM for 2010-2011 Ph.D. Fellowship support.

\section{References}

[1] M. Turner, D. Budgen, and P. Brereton. Turning Software into a service. Computer. vol. 36 , no. 10, pp. 38-44, 2003.

[2] http://www.platformasaservice.com/

[3] The Cloudscaling Team. Infrastructure-as-a-servicebuilders-guide-v1-0

[4] W. Emeneker, et al, Dynamic Virtual Clustering with Xen and Moab, International Symposium on Parallel and Distributed Processing with Applications (ISPA), Springer-Verlag LNCS 4331, 2006, pp. 440-451

[5] VMware ESX Server, VMware Inc. http://www.vmware.com/products/vi/esx/

[6] Xen Hypervisor, http://www.xen.org/

[7] S. Li, and D. Tirupati. Technology choice with stochastic demands and dynamic capacity allocation:
A two-product analysis. Journal of Operations Management, Vol. 12, no 3-4, pp. 239-258, 1995.

[8] Y. Wu, K. Hwang, Y. Yuan, and W. Zheng, "Adaptive Workload Prediction of Grid Performance in Confidence Windows", IEEE Trans. on Parallel and Distributed Systems, to appear, (on-line published Aug. 14, 2009)

[9] K. Hoffman. How to Build Your First Azure-Powered MVC App. http://cloudcomputing.syscon.com/node/1124872

[10] L. Kleinrock, Queueing Systems, Volume 2: Computer Applications. John Wiley and Sons, Inc., 1976.

[11] L. Slothouber. A model of web server performance. In Proceedings of the 5th International World Wide Web Conference (WWW). Paris, France. 1996.

[12] J. Chase, and R. Doyle. Balance of power: Energy management for server clusters. In Proceedings of the 8th Workshop on Hot Topics in Operating Systems (HotOS-VIII). Elmau, Germany. 2001.

[13] B. Urgaonkar, and P. Shenoy, Cataclysm: Handling extreme overloads in internet services. In Proceedings of the 23rd Annual ACM SIGACT-SIGOPS Symposium on Principles of Distributed Computing (PODC'04). St. John's, Newfoundland, Canada. 2004.

[14] R. Levy, J. Nagarajarao, G. Pacifici, M. Spreitzer, A. Tantawi, and A. Youssef. Performance management for cluster based web services. In IFIP/IEEE 8th International Symposium on Integrated Network Management. Vol. 246, pp. 247-261. 2003.

[15] D. Menasce, Web server software architectures. IEEE Internet Computing. Vol. 7 no. 6, 2003.

[16] D. Villela , P. Pradhan, and D. Rubenstein. Provisioning servers in the application tier for ecommerce systems. ACM Transactions on Internet Technology (TOIT). Vol. 7, no. 1, 2007.

[17] S. Ranjan, J. Rolia, H. FU, and E. Knightly. QoSdriven servermigration for internet data centers. In Proceedings of the 10th International Workshop on Quality of Service(IWQoS), Miami, FL. 2002.

[18] A. Kamra, V. Misra, E.M. Nahum, Yaksha: a selftuning controller for managing the performance of 3tiered Web sites, In Proceedings of the 12th International Workshop on Quality of Service(IWQoS), Passau, Germany, 2004.

[19] B. Urgaonkar, G. Pacifici, P. Shenoy, M.Spreitzer, and A. Tantawi. Analytic Modeling of Multi-tier Internet Services and its Applications. ACM Transactions on the Web (TWEB 2007), Vol. 1, No. 1, pp. 1-35, May 2007.

[20] B. Urgaonkar, P. Shenoy, A. Chandra, P. Goyal, and T. Wood. Agile Dynamic Provisioning of Multi-tier Internet Applications. ACM Transactions on Adaptive and Autonomous Systems (TAAS), Vol. 3, No. 1, pp. 1-39, March 2008.

[21] M. Armbrust, A. Fox, R. Griffith, A. D. Joseph, R. H. Katz, A. Konwinski, G. Lee, D. A. Patterson, A. 
Rabkin, I. Stoica, and M. Zaharia. Above the clouds: A berkeley view of cloud computing. Technical Report No. UCB/EECS-2009-28

[22] R. Buyya, C. S. Yeo, and S. Venugopal, MarketOriented Cloud Computing: Vision, Hype, and Reality for Delivering IT Services as Computing Utilities, Proceedings of the 10th IEEE International Conference on High Performance Computing and Communications (HPCC 2008, IEEE CS Press, Los Alamitos, CA, USA), Sept. 25-27, 2008, Dalian, China. [23] http://aws.amazon.com/ec2/

[24] http://www.eucalyptus.com/

[25] http://rubis.ow2.org/

[26] M. Arlitt, and T. Jin. Workload Characterization of the 1998 World Cup Web Site. Tech. Rep. HPL-199935R1, HP Labs. 1999

[27] R. N. Calheiros, R. Ranjan, A. Beloglazov, C. Rose, and R. Buyya, CloudSim: A Toolkit for Modeling and Simulation of Cloud Computing Environments and Evaluation of Resource Provisioning Algorithms, Software: Practice and Experience, ISSN: 0038-0644, Wiley Press, New York, USA, 2010.

\section{Biographical Sketches:}

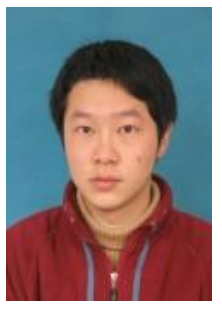

Fan Zhang received the B.S. in computer science from Hubei Univ. of Technology and M. S. in control science and engineering from Huazhong University of Science and technology. He is currently a Ph.D. student in Department of Automation, Tsinghua University, Beijing, China. His research interests include data center networks and grid/cloud computing. Contact him at: zhang-fan07@mails.tsinghua.edu.cn

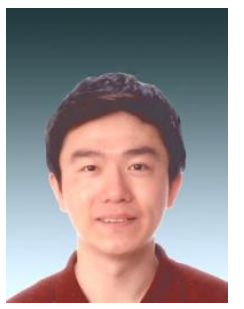

Junwei Cao is currently Professor and Assistant Dean, Research Institute of Information Technology, Tsinghua University, China. He was a Research Scientist at MIT LIGO Laboratory and NEC Laboratories Europe. He received the $\mathrm{PhD}$ in Computer Science from University of Warwick, UK, in 2001. He is a Senior Member of the IEEE Computer Society and a Member of the ACM and CCF. Contact him via Email: jcao@tsinghua.edu.cn

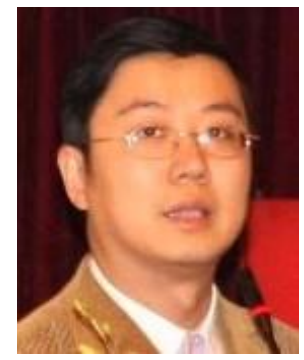

Hong Cai is currently a senior software engineer of IBM China development lab and chair of TECGC (Technical Expert Council Greater China). He received the B.S and Ph.D. from Tsinghua Univ., China. His research interests include dynamic resource provisioning, distributed computing, etc. Contact him via Email: caihong@cn.ibm.com

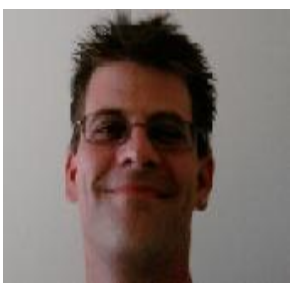

James J. Mulcahy is currently a $\mathrm{Ph} . \mathrm{D}$ student in Computer Science at Florida Atlantic University in Boca Raton, Florida, USA. His research interests include grid/cloud applications, software engineering and re-engineering of legacy systems. He has more than 20 years of business experience in developing and evolving large supply chain and e-commerce software applications. James is a member of IEEE and the Association of Computing Machinery (ACM). James can be reached via email at jmulcah1@fau.edu

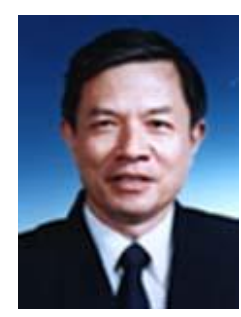

Cheng Wu is a Professor of Department of Automation, Tsinghua Univ. China, Director of National CIMS Engineering Research Center, and Member of Chinese Academy of Engineering. He received his B.S. and M.S. from Department of automation, Tsinghua Univ. in 1962 and 1966 respectively. His research interests include complex manufacturing system scheduling, grid/cloud applications, etc. Contact him via Email: wuc@tsinghua.edu.cn 